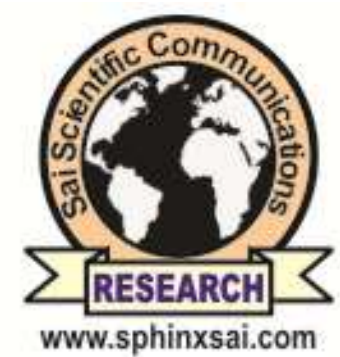

International Journal of PharmTech Research CODEN (USA): IJPRIF, ISSN: 0974-4304, ISSN(Online): 2455-9563

Vol.12, No.01, pp 28-36

2019

\title{
Assessment of Occupational Electromagnetic Fields exposure for selected Electrotherapeutic modality
}

\author{
Maher El-Keblawy ${ }^{1}$, Abeer Mahmoud Yousef ${ }^{2 \star}$, Mahmoud Elqazaz ${ }^{3}$ \\ ${ }^{1}$ Professor, Faculty of Physical Therapy, Cairo University, Cairo, Egypt. \\ ${ }^{2}$ Lecture, Faculty of Physical Therapy, Cairo University, Cairo, Egypt. \\ ${ }^{3}$ The Department of Physical Therapy *, Samanoud Central Hospital, El-Gharbeya, \\ Egypt.
}

\begin{abstract}
Purpose of the study: The main aim of this study was to investigate the physical therapists' occupational exposure to electromagnetic fields surrounding electrotherapy appliances with different techniques of application and comparing the results with the reference limits set by international organizations to provide the appropriate advice and guidelines for safe limits of exposure. Furthermore, the second main objective was to investigate the effect of equipment grounding, as one of protection designs, on the amount of electromagnetic field exposure.Materials and methods:- In the present study, electric field intensity was measured by Nared EMR-200 and magnetic flux density (G) by Tesla/ Gauss meter and they were measured in the three planes around the electrotherpay device (phyaction 787 series) under both ungrounded and grounded conditions. The same parameters were also determined for the electrodes that were put on water phantom, and on the cables that were connected the device to the electrodes. Results:- Comparing the obtained results with the safe limits set by International Committee for Non-Ionizing Radiation Protection revealed that the electric field intensities at the different measured points were within the safe limits. However, the magnetic flux density measured at the electrodes was above these limits. Conclusion:- The present assessment sheds more light on the occupational electromagnetic exposure for physiotherapist and recommends for more safety procedures in treatment with this modality.
\end{abstract}

Keywords : Electromagnetic; Interferential; Diadynamic; International Committee for NonIonizing Radiation Protection; Hazard.

\section{Introduction}

Interferential electrotherapy (IFE) is a common physiotherapeutic treatment modality. Its high carrier frequency (around $4000 \mathrm{~Hz}$ ) produces lower impedance to the skin and allows deeper penetration into the tissue $^{1}$. The application may be either quadripolarin which two circuits are involved; four electrodes are usually used $^{2}$. The maximum interference effect takes place near the center, with the field gradually decreasing in 
strength as it moves toward the periphery ${ }^{3}$. The electrodes are placed in a coplanar arrangement to treat a flat surface such as the back. It is normally recommended to use the largest electrode sizes that can conveniently be applied in order to ensure a comfortable current of sufficient intensity throughout the treated area ${ }^{4}$. The other application is the bipolar (pre-modulated) technique in which the two medium-frequency currents are superimposed within the machine so that the single current interference occurs throughout the region between the two electrodes ${ }^{5}$.With bipolar interferential, however, since the current is being burst inside the unit itself, numbness does not occur and a larger treatment area is established with the actual therapeutic frequency ${ }^{6}$.Diadynamic currents are alternate currents rectified in complete or half waves, with frequency of 50 and $100 \mathrm{~Hz}^{7}$. Stimulation of thin fibers as well can only be obtained at higher current amplitudes ${ }^{8}$.The guidelines set by ICNIRP and other organizations aimed to protect against maximum external field exposure and maximum internal induced currents ${ }^{9}$, It is conceivable that daily exposure time may be relevant to the potential chronic effects of the fields ${ }^{\mathbf{1 0}}$.Protection of workers could be achieved through engineering controls including a redesign of equipment or work processes and/or isolation of the hazard. equipment Personal protective suits are available to screen the user from high ambient field exposures. These garments are constructed from conductive fabrics and can provide a substantial faraday cage shielding effect, but only if the user is fully enclosed in the suit ${ }^{11}$.

\section{Experimental:}

This study was applied in the faculty of physical therapy, Cairo university, Cairo, Egypt and during the period between March 2015 to August 2015.The study was delimited to the following currents:-1-Interferential current which was investigated according the following parameters: main frequency of $4 \mathrm{KHz}$, beat frequency of $100 \mathrm{~Hz}$ and current intensity (amplitude) of $50 \mathrm{~mA}$

2- Diadynamic which was investigated in the following parameters: Main frequency of $50 \mathrm{~Hz}$, Current intensity (amplitude) of $50 \mathrm{~mA}$.

\section{Instruments:}

Phyaction 787 series (Fig 1); manufactured in the Netherlands by Uniphy BV; was used as an experimental instrument which can produce Interferential and Diadynamiccurrrents. Electric filed intensity was measured by electric field meter (Narda, manufactured by Wandel\&Goltermann, Germany, has a frequency range of $5 \mathrm{~Hz}-100 \mathrm{KHz}$, and electric field intensity range of $0.01-100 \mathrm{KV} / \mathrm{m}$ ). Magnetic flux density was measured by a hand-held Gauss/Tesla meter(model 4080, with probe type T-4048.001 manufactured by FW Bell in U.S.A, magnetic flux density in Gauss, from $0.1 \mathrm{G}$ up to $200 \mathrm{G}$ ).

\section{Conduction of measurement:}

The measurements of both electric and magnetic fields around the apparatus working for interferential current mode generated from a beat oscillator were conducted with the following parameters: main frequency of $4 \mathrm{KHz}$, beat frequency of $100 \mathrm{~Hz}$ and current intensity (amplitude) of $50 \mathrm{~mA}$. Interferential currents were applied by four carbon-rubber electrodes with conducting gel and were held by straps over a water bag i.e. quadripolar technique. The four electrodes were arranged in a cross arrangement around a rubber bag full of water act as a patient phantom (Fig 2). The measurements of both electric and magnetic fields around the apparatus working for diadynamic current Monophase Fixe (MF) mode generated from a beat oscillator were conducted with the following parameters: Main frequency of $50 \mathrm{~Hz}$, Current intensity (amplitude) of $50 \mathrm{~mA}$. Diadynamic current in MF mode was applied by two carbon-rubber electrodes with conducting gel and were held by straps over the water bag.Exposure assessment was carried out by setting azero point on the surface of the device and on the water phantom to be used as reference points. The field was determined in $\mathrm{X}, \mathrm{Y}$ and $\mathrm{Z}$ planes from the device and the electrodes and ata point in the middle of cables. Device grounding was accomplished by connecting its body to the metallic ground object through a wide connecting wire.The measurements of both electric and magnetic fields strength were done in the near field atthe close vicinity of the setup's three components (device, cable, and electrodes) and at the far field at 1 meter (safe distance set by ICRNIP) away from the set up components. The values of the electric and magnetic field strength around the apparatus working at the different modes of operation were measured and tabulated. Each measurement was repeated three times and the average of each was considered. 


\section{Results}

As presented at table (1), the correlations between distance and the mean value of electrical and magnetic fields around the interferential bipolar device at different intensities and axes at earthed and unearthed were studied through the Pearson product moment correlation coefficient.

Table (1):The statistical analysis of (Correlation between Distance and electrical or magnetic current in Interferential bipolar device) in both Earthed and Un Earthed conditions:

\begin{tabular}{|c|c|c|c|c|c|c|c|}
\hline & \multirow[b]{2}{*}{ Intensity } & \multirow[b]{2}{*}{ Axis } & \multirow[b]{2}{*}{ Current } & \multicolumn{2}{|l|}{ Earthed } & \multicolumn{2}{|l|}{ Un-Earthed } \\
\hline & & & & $\begin{array}{l}\text { Pearson } \\
\text { Correlation }\end{array}$ & P-value & $\begin{array}{l}\text { Pearson } \\
\text { Correlation }\end{array}$ & P-value \\
\hline \multirow{18}{*}{ Device } & \multirow{6}{*}{$10 \mathrm{~mA}$} & \multirow{2}{*}{$\mathrm{X}$ axis } & Electrical & -0.626 & $0.039 *$ & -0.832 & $0.002 *$ \\
\hline & & & Magnetic & $\begin{array}{l}-0.808 \\
\end{array}$ & $0.003 *$ & -0.563 & 0.071 \\
\hline & & \multirow{2}{*}{$\mathrm{Y}$ axis } & Electrical & -0.893 & $0.000 *$ & -0.733 & $0.010^{*}$ \\
\hline & & & Magnetic & -0.697 & $0.017 *$ & -0.208 & 0.539 \\
\hline & & \multirow[t]{2}{*}{$\mathrm{Z}$ axis } & Electrical & -0.692 & 0.018 & -0.748 & $0.008^{*}$ \\
\hline & & & Magnetic & -0.500 & 0.117 & -0.910 & $0.000^{*}$ \\
\hline & \multirow{6}{*}{$50 \mathrm{~mA}$} & \multirow{2}{*}{$\mathrm{X}$ axis } & Electrical & -0.738 & $0.010 *$ & -0.831 & $0.002 *$ \\
\hline & & & Magnetic & -0.758 & $0.007 *$ & -0.474 & 0.141 \\
\hline & & \multirow{2}{*}{$\mathrm{Y}$ axis } & Electrical & -0.943 & $0.000^{*}$ & -0.794 & $0.003 *$ \\
\hline & & & Magnetic & -0.907 & $0.000^{*}$ & -0.525 & 0.097 \\
\hline & & \multirow[t]{2}{*}{$\mathrm{Z}$ axis } & Electrical & -0.874 & $0.000^{*}$ & -0.579 & 0.062 \\
\hline & & & Magnetic & -0.622 & $0.041^{*}$ & -0.688 & $0.019 *$ \\
\hline & \multirow{6}{*}{$100 \mathrm{~mA}$} & \multirow{2}{*}{$\mathrm{X}$ axis } & Electrical & -0.909 & $0.000 *$ & -0.835 & $0.001 *$ \\
\hline & & & Magnetic & -0.566 & 0.069 & -0.228 & 0.501 \\
\hline & & \multirow{2}{*}{$\mathrm{Y}$ axis } & Electrical & -0.866 & $0.001 *$ & -0.668 & $0.025^{*}$ \\
\hline & & & Magnetic & -0.764 & $0.006^{*}$ & -0.178 & 0.600 \\
\hline & & \multirow[t]{2}{*}{$\mathrm{Z}$ axis } & Electrical & -0.610 & $0.046^{*}$ & -0.246 & 0.466 \\
\hline & & & Magnetic & -0.712 & $0.014 *$ & -0.762 & $0.006^{*}$ \\
\hline
\end{tabular}

* Significant correlation P-value $<0.05$

As presented at table (2), Considering earthed, it revealed that there was negative strong significant correlation between distance and electrical and magnetic fields $(\mathrm{p}<0.05)$ in all intensities at all axes except for magnetic fields at $X$ and $Z$ axes in $10 \mathrm{~mA}$ and at $Y$ axis in $50 \mathrm{~mA}$ and at $\mathrm{X}$ axis in $100 \mathrm{~mA}$. Regarding unearthed it revealed that there was negative strong significant correlation between (distance and electrical) and (distance and magnetic fields) ( $\mathrm{p}<0.05)$ in $\mathrm{X}, \mathrm{Y}$ and $\mathrm{Z}$ axes at $10 \mathrm{~mA}, 50 \mathrm{~mA}$ and100 $\mathrm{mA}$.

Table (2):The statistical analysis of (Correlation between Distance and electrical or magnetic current in Interferential bipolar electrodes) in both Earthed and Un Earthed conditions:

\begin{tabular}{|c|c|c|c|c|c|c|c|}
\hline & \multirow[b]{2}{*}{ Intensity } & \multirow[b]{2}{*}{ Axis } & \multirow[b]{2}{*}{ Current } & \multicolumn{2}{|l|}{ Earthed } & \multicolumn{2}{|l|}{ Un-Earthed } \\
\hline & & & & $\begin{array}{l}\text { Pearson } \\
\text { Correlation }\end{array}$ & P-value & $\begin{array}{l}\text { Pearson } \\
\text { Correlation }\end{array}$ & P-value \\
\hline \multirow{11}{*}{ Electrodes } & \multirow{6}{*}{$10 \mathrm{~mA}$} & \multirow{2}{*}{$\mathrm{X}$ axis } & Electrical & -0.803 & $0.003^{*}$ & -0.618 & $0.043^{*}$ \\
\hline & & & Magnetic & -0.484 & 0.131 & -0.772 & $0.005^{*}$ \\
\hline & & \multirow{2}{*}{ Y axis } & Electrical & -0.755 & $0.007 *$ & -0.902 & $0.000 *$ \\
\hline & & & Magnetic & -0.753 & $0.007 *$ & -0.276 & 0.412 \\
\hline & & \multirow[t]{2}{*}{$\mathrm{Z}$ axis } & Electrical & -0.766 & $0.006^{*}$ & -0.788 & $0.004 *$ \\
\hline & & & Magnetic & -0.376 & 0.254 & -0.475 & 0.140 \\
\hline & \multirow{5}{*}{$50 \mathrm{~mA}$} & \multirow{2}{*}{$\mathrm{X}$ axis } & Electrical & -0.911 & $0.000 *$ & -0.853 & $0.001 *$ \\
\hline & & & Magnetic & -0.658 & $0.028 *$ & -0.552 & 0.078 \\
\hline & & \multirow{2}{*}{ Y axis } & Electrical & -0.846 & $0.001 *$ & -0.697 & $0.017 *$ \\
\hline & & & Magnetic & -0.602 & 0.050 & -0.982 & $0.000 *$ \\
\hline & & $\mathrm{Z}$ axis & Electrical & -0.805 & $0.003^{*}$ & -0.479 & 0.136 \\
\hline
\end{tabular}




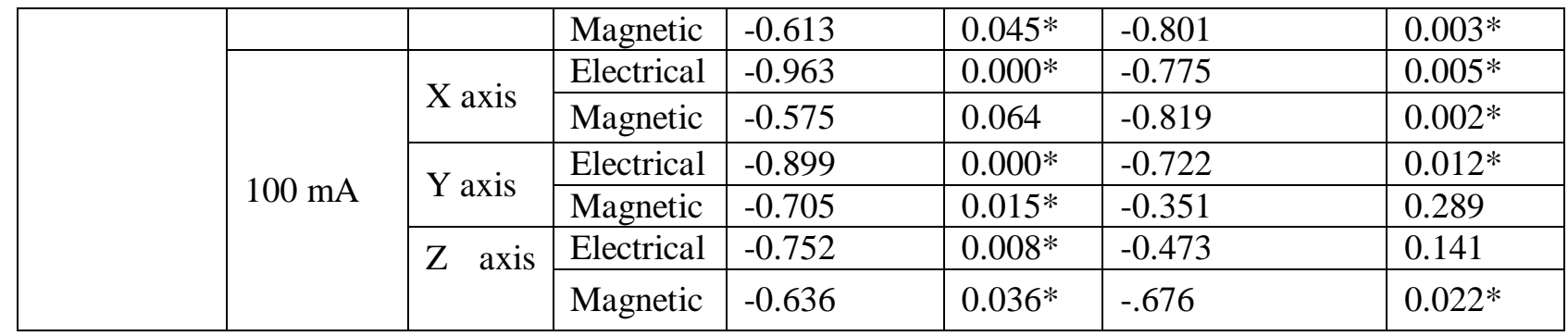

* Significant correlation P-value $<0.05$

As presented at table (3), the correlations between distance and the mean value of electrical and magnetic fields around the interferential quadripolar device at different intensities and axes at earthed and unearthed were studied through the Pearson product moment correlation coefficient. Considering earthed and UN earthedit revealed that there was negative strong significant correlation between distance and electrical and magnetic fields $(\mathrm{p}<0.05)$ in all intensities at all axes.

Table (3):The statistical analysis of (Correlation between Distance and electrical or magnetic current in Interferential quadripolar device) in both Earthed and Un Earthed conditions:

\begin{tabular}{|c|c|c|c|c|c|c|c|}
\hline & \multirow[b]{2}{*}{ Intensity } & \multirow[b]{2}{*}{ Axis } & \multirow[b]{2}{*}{ Current } & \multicolumn{2}{|l|}{ Earthed } & \multicolumn{2}{|l|}{ Un-Earthed } \\
\hline & & & & $\begin{array}{l}\text { Pearson } \\
\text { Correlation }\end{array}$ & P-value & $\begin{array}{l}\text { Pearson } \\
\text { Correlation }\end{array}$ & P-value \\
\hline \multirow{18}{*}{ Device } & \multirow{6}{*}{$10 \mathrm{~mA}$} & \multirow{2}{*}{$\mathrm{X}$ axis } & Electrical & -0.844 & $0.001 *$ & -0.838 & $0.001^{*}$ \\
\hline & & & Magnetic & -0.663 & $0.026^{*}$ & -0.259 & 0.442 \\
\hline & & \multirow{2}{*}{ Y axis } & Electrical & -0.747 & $0.008^{*}$ & -0.139 & 0.683 \\
\hline & & & Magnetic & -0.787 & $0.004 *$ & -0.244 & 0.471 \\
\hline & & \multirow[t]{2}{*}{$\mathrm{Z}$ axis } & Electrical & -0.806 & $0.003^{*}$ & -0.378 & 0.252 \\
\hline & & & Magnetic & -0.728 & $0.011^{*}$ & -0.748 & $0.008^{*}$ \\
\hline & \multirow{6}{*}{$50 \mathrm{~mA}$} & \multirow{2}{*}{$\mathrm{X}$ axis } & Electrical & -0.837 & $0.001 *$ & -0.680 & $0.021^{*}$ \\
\hline & & & Magnetic & -0.879 & $0.000^{*}$ & -0.547 & 0.082 \\
\hline & & \multirow{2}{*}{$Y$ axis } & Electrical & -0.632 & $0.037 *$ & 0.422 & 0.196 \\
\hline & & & Magnetic & -0.830 & $0.002 *$ & -0.187 & 0.582 \\
\hline & & \multirow[t]{2}{*}{$\mathrm{Z}$ axis } & Electrical & -0.797 & $0.003^{*}$ & -0.424 & 0.194 \\
\hline & & & Magnetic & -0.861 & $0.001 *$ & -0.228 & 0.500 \\
\hline & \multirow{6}{*}{$100 \mathrm{~mA}$} & \multirow{2}{*}{$\mathrm{X}$ axis } & Electrical & -0.846 & $0.001^{*}$ & -0.849 & $0.001 *$ \\
\hline & & & Magnetic & -0.840 & $0.001 *$ & -0.722 & $0.012^{*}$ \\
\hline & & \multirow{2}{*}{$\mathrm{Y}$ axis } & Electrical & -0.808 & $0.003^{*}$ & -0.452 & 0.163 \\
\hline & & & Magnetic & -0.802 & $0.003^{*}$ & -0.391 & 0.234 \\
\hline & & \multirow[t]{2}{*}{$\mathrm{Z}$ axis } & Electrical & -0.758 & $0.007 *$ & -0.358 & 0.280 \\
\hline & & & Magnetic & -0.683 & $0.021^{*}$ & -0.309 & 0.355 \\
\hline
\end{tabular}

* Significant correlation P-value $<0.05$

As presented at table (4), the correlations between distance and the mean value of electrical and magnetic fields around the interferential quadripolar electrodes at different intensities and axes at earthed and un-earthed were studied through the Pearson product moment correlation coefficient. 
Table (4):The statistical analysis of (Correlation between Distance and electrical or magnetic current in Interferential quadripolar electrodes) in both Earthed and Un Earthed conditions:

\begin{tabular}{|c|c|c|c|c|c|c|c|}
\hline & \multirow[b]{2}{*}{ Intensity } & \multirow[b]{2}{*}{ Axis } & \multirow[b]{2}{*}{ Current } & \multicolumn{2}{|l|}{ Earthed } & \multicolumn{2}{|l|}{ Un-Earthed } \\
\hline & & & & $\begin{array}{l}\text { Pearson } \\
\text { Correlation }\end{array}$ & P-value & $\begin{array}{l}\text { Pearson } \\
\text { Correlation }\end{array}$ & P-value \\
\hline \multirow{18}{*}{ Electrodes } & \multirow{6}{*}{$10 \mathrm{~mA}$} & \multirow{2}{*}{$\mathrm{X}$ axis } & Electrical & -0.913 & $0.000 *$ & -0.870 & $0.001 *$ \\
\hline & & & Magnetic & -0.673 & $0.023^{*}$ & -0.835 & $0.001 *$ \\
\hline & & \multirow{2}{*}{ Y axis } & Electrical & -0.840 & $0.001 *$ & -0.263 & 0.435 \\
\hline & & & Magnetic & -0.814 & $0.002 *$ & -0.829 & $0.002 *$ \\
\hline & & \multirow[t]{2}{*}{$\mathrm{Z}$ axis } & Electrical & -0.979 & $0.000 *$ & 0.824 & $0.002 *$ \\
\hline & & & Magnetic & -0.883 & $0.000 *$ & -0.864 & $0.001 *$ \\
\hline & \multirow{6}{*}{$50 \mathrm{~mA}$} & \multirow{2}{*}{$\mathrm{X}$ axis } & Electrical & -0.808 & $0.003^{*}$ & -0.840 & $0.001 *$ \\
\hline & & & Magnetic & -0.506 & 0.113 & -0.697 & $0.017 *$ \\
\hline & & \multirow{2}{*}{ Y axis } & Electrical & -0.749 & $0.008^{*}$ & -0.737 & $0.010 *$ \\
\hline & & & Magnetic & -0.701 & $0.016^{*}$ & -0.635 & $0.036^{*}$ \\
\hline & & \multirow[t]{2}{*}{$\mathrm{Z}$ axis } & Electrical & -0.697 & $0.017^{*}$ & 0.503 & 0.115 \\
\hline & & & Magnetic & -0.859 & $0.001 *$ & -0.729 & $0.011 *$ \\
\hline & \multirow{6}{*}{$100 \mathrm{~mA}$} & \multirow{2}{*}{$\mathrm{X}$ axis } & Electrical & -0.835 & $0.001 *$ & -0.842 & $0.001 *$ \\
\hline & & & Magnetic & -0.841 & $0.001 *$ & -0.814 & $0.002 *$ \\
\hline & & \multirow{2}{*}{$\mathrm{Y}$ axis } & Electrical & -0.782 & $0.004 *$ & -0.824 & $0.002 *$ \\
\hline & & & Magnetic & -0.803 & $0.003 *$ & -0.842 & $0.001 *$ \\
\hline & & \multirow{2}{*}{$\mathrm{Z}$ axis } & Electrical & -0.747 & $0.008^{*}$ & 0.026 & 0.940 \\
\hline & & & Magnetic & -0.709 & $0.014^{*}$ & -0.620 & $0.042 *$ \\
\hline
\end{tabular}

* Significant correlation P-value $<0.05$

As presented at table (5), the correlations between distance and the mean value of electrical and magnetic fields around the Diadynamic DF device at different intensities and axes at earthed and un-earthed were studied through the Pearson product moment correlation coefficient.

Table (5):The statistical analysis of (Correlation between Distance and electrical or magnetic current in Diadynamic DF device) in both Earthed and Un Earthed conditions:

\begin{tabular}{|c|c|c|c|c|c|c|c|}
\hline & \multirow[b]{2}{*}{ Intensity } & \multirow[b]{2}{*}{ Axis } & \multirow[b]{2}{*}{ Current } & \multicolumn{2}{|l|}{ Earthed } & \multicolumn{2}{|l|}{ Un-Earthed } \\
\hline & & & & $\begin{array}{l}\text { Pearson } \\
\text { Correlation }\end{array}$ & P-value & $\begin{array}{l}\text { Pearson } \\
\text { Correlation }\end{array}$ & P-value \\
\hline \multirow{18}{*}{ Device } & \multirow{6}{*}{$10 \mathrm{~mA}$} & \multirow{2}{*}{$\mathrm{X}$ axis } & Electrical & -0.762 & $0.006^{*}$ & -0.823 & $0.002 *$ \\
\hline & & & Magnetic & -0.504 & 0.114 & -0.735 & $0.010^{*}$ \\
\hline & & \multirow{2}{*}{$\mathrm{Y}$ axis } & Electrical & -0.772 & $0.005^{*}$ & -0.379 & 0.250 \\
\hline & & & Magnetic & -0.729 & $0.011^{*}$ & -.671 & $0.024 *$ \\
\hline & & \multirow[t]{2}{*}{\begin{tabular}{|ll}
$\mathrm{Z}$ & axis \\
\end{tabular}} & Electrical & -0.784 & 0.004* & -0.581 & 0.061 \\
\hline & & & Magnetic & -0.762 & $0.006^{*}$ & -0.466 & 0.149 \\
\hline & \multirow{6}{*}{$50 \mathrm{~mA}$} & \multirow{2}{*}{$\mathrm{X}$ axis } & Electrical & -0.871 & $0.000^{*}$ & -.874 & $0.000^{*}$ \\
\hline & & & Magnetic & -0.794 & $0.004 *$ & -0.394 & 0.230 \\
\hline & & \multirow{2}{*}{ Y axis } & Electrical & -0.662 & $0.027 *$ & 0.017 & 0.960 \\
\hline & & & Magnetic & -0.675 & $0.023^{*}$ & -0.677 & $0.022 *$ \\
\hline & & \multirow[t]{2}{*}{$\begin{array}{ll}\mathrm{Z} \text { axis } \\
\end{array}$} & Electrical & -0.613 & $0.045^{*}$ & -0.607 & $0.048^{*}$ \\
\hline & & & Magnetic & -0.420 & 0.199 & -0.949 & $0.000^{*}$ \\
\hline & \multirow{6}{*}{$80 \mathrm{~mA}$} & \multirow[b]{2}{*}{$\mathrm{X}$ axis } & Electrical & -0.892 & $0.000^{*}$ & -0.833 & $0.001 *$ \\
\hline & & & Magnetic & -0.915 & $0.000^{*}$ & -0.501 & 0.116 \\
\hline & & \multirow{2}{*}{$\mathrm{Y}$ axis } & Electrical & -0.843 & $0.001^{*}$ & -0.474 & 0.141 \\
\hline & & & Magnetic & -0.698 & $0.017^{*}$ & -0.231 & 0.495 \\
\hline & & \multirow[t]{2}{*}{$\mathrm{Z}$ axis } & Electrical & -0.827 & $0.002 *$ & -0.168 & 0.621 \\
\hline & & & Magnetic & -0.789 & $0.004^{*}$ & -0.285 & 0.395 \\
\hline
\end{tabular}

* Significant correlation P-value $<0.05$ 
As presented at table (6), the correlations between distance and the mean value of electrical and magnetic fields around the Diadynamic DF electrodes at different intensities and axes at earthed and unearthed were studied through the Pearson product moment correlation coefficient.

Table (6): The statistical analysis of (Correlation between Distance and electrical or magnetic current in DiadynamicDF electrodes) in both Earthed and Un Earthed conditions:

\begin{tabular}{|c|c|c|c|c|c|c|c|}
\hline & \multirow[b]{2}{*}{ Intensity } & \multirow[b]{2}{*}{ Axis } & \multirow[b]{2}{*}{ Current } & \multicolumn{2}{|l|}{ Earthed } & \multicolumn{2}{|l|}{ Un-Earthed } \\
\hline & & & & $\begin{array}{l}\text { Pearson } \\
\text { Correlation }\end{array}$ & P-value & $\begin{array}{l}\text { Pearson } \\
\text { Correlation }\end{array}$ & P-value \\
\hline \multirow{18}{*}{ Electrodes } & \multirow{6}{*}{$10 \mathrm{~mA}$} & \multirow{2}{*}{$\mathrm{X}$ axis } & Electrical & -0.817 & $0.002 *$ & 0.864 & $0.001^{*}$ \\
\hline & & & Magnetic & $\begin{array}{l}-0.650 \\
\end{array}$ & $0.030 *$ & -0.727 & $0.011^{*}$ \\
\hline & & \multirow{2}{*}{ Y axis } & Electrical & -0.813 & $0.002 *$ & -0.622 & $0.041^{*}$ \\
\hline & & & Magnetic & -0.631 & $0.037 *$ & -0.611 & $0.046^{*}$ \\
\hline & & \multirow[t]{2}{*}{$\mathrm{Z}$ axis } & Electrical & -0.844 & $0.001 *$ & -0.190 & 0.577 \\
\hline & & & Magnetic & -0.37 & 0.262 & -0.845 & $0.001^{*}$ \\
\hline & \multirow{6}{*}{$50 \mathrm{~mA}$} & \multirow{2}{*}{$\mathrm{X}$ axis } & Electrical & -0.920 & $0.000^{*}$ & -0.852 & $0.001^{*}$ \\
\hline & & & Magnetic & -0.860 & $0.001 *$ & -0.812 & $0.002 *$ \\
\hline & & \multirow{2}{*}{$\mathrm{Y}$ axis } & Electrical & -0.865 & $0.001 *$ & -0.702 & $0.016^{*}$ \\
\hline & & & Magnetic & -0.737 & $0.010^{*}$ & -.676 & $0.022^{*}$ \\
\hline & & \multirow[t]{2}{*}{$\mathrm{Z}$ axis } & Electrical & -0.866 & $0.001 *$ & -0.108 & 0.753 \\
\hline & & & Magnetic & -0.65 & $0.028^{*}$ & -0.801 & $0.003^{*}$ \\
\hline & \multirow{6}{*}{$80 \mathrm{~mA}$} & \multirow{2}{*}{$\mathrm{X}$ axis } & Electrical & -0.956 & $0.000 *$ & -0.843 & $0.001^{*}$ \\
\hline & & & Magnetic & -0.700 & $0.016^{*}$ & -0.542 & 0.085 \\
\hline & & \multirow{2}{*}{$\mathrm{Y}$ axis } & Electrical & -0.856 & $0.001 *$ & -0.685 & $0.020^{*}$ \\
\hline & & & Magnetic & -0.727 & $0.011^{*}$ & -0.944 & $0.000^{*}$ \\
\hline & & \multirow[t]{2}{*}{$\mathrm{Z}$ axis } & Electrical & -0.953 & $0.000 *$ & -0.243 & 0.471 \\
\hline & & & Magnetic & -0.700 & $0.016^{*}$ & -0.940 & $0.000^{*}$ \\
\hline
\end{tabular}

* Significant correlation P-value $<0.05$

As presented at table (7), the correlations between distance and the mean value of electrical and magnetic fields around the Diadynamic MF device at different intensities and axes at earthed and un-earthed were studied through the Pearson product moment correlation coefficient.

Table (7): The statistical analysis of (Correlation between Distance and electrical or magnetic current in DiadynamicMF device) in both Earthed and Un Earthed conditions:

\begin{tabular}{|c|c|c|c|c|c|c|c|}
\hline & \multirow[b]{2}{*}{ Intensity } & \multirow[b]{2}{*}{ Axis } & \multirow[b]{2}{*}{ Current } & \multicolumn{2}{|l|}{ Earthed } & \multicolumn{2}{|l|}{ Un-Earthed } \\
\hline & & & & \begin{tabular}{|l|} 
Pearson \\
Correlation
\end{tabular} & P-value & $\begin{array}{l}\text { Pearson } \\
\text { Correlation }\end{array}$ & P-value \\
\hline \multirow{16}{*}{ Device } & \multirow{6}{*}{$10 \mathrm{~mA}$} & \multirow{2}{*}{$\mathrm{X}$ axis } & \begin{tabular}{|l|} 
Electrical \\
\end{tabular} & -0.856 & \begin{tabular}{|l|}
$0.001^{*}$ \\
\end{tabular} & -0.842 & $0.001 *$ \\
\hline & & & Magnetic & -0.713 & $0.014^{*}$ & -0.450 & 0.165 \\
\hline & & \multirow{2}{*}{$\mathrm{Y}$ axis } & Electrical & -0.746 & $0.008^{*}$ & -0.633 & $0.036^{*}$ \\
\hline & & & Magnetic & -0.731 & $0.011^{*}$ & -0.360 & 0.277 \\
\hline & & \multirow[t]{2}{*}{$\mathrm{Z}$ axis } & Electrical & -0.721 & $0.012^{*}$ & -0.486 & 0.130 \\
\hline & & & Magnetic & -0.831 & $0.002^{*}$ & -0.829 & $0.002 *$ \\
\hline & \multirow{6}{*}{$50 \mathrm{~mA}$} & \multirow{2}{*}{$\mathrm{X}$ axis } & Electrical & -0.936 & $0.000^{*}$ & -0.824 & $0.002 *$ \\
\hline & & & Magnetic & -0.853 & $0.001^{*}$ & -0.708 & $0.015^{*}$ \\
\hline & & \multirow{2}{*}{ Y axis } & Electrical & -0.862 & $0.001^{*}$ & -0.764 & $0.006^{*}$ \\
\hline & & & Magnetic & -0.671 & \begin{tabular}{|l|}
$0.024^{*}$ \\
\end{tabular} & -0.147 & 0.666 \\
\hline & & \multirow[t]{2}{*}{\begin{tabular}{|l}
$\mathrm{Z}$ axis \\
\end{tabular}} & Electrical & -0.697 & \begin{tabular}{|l|}
$0.017^{*}$ \\
\end{tabular} & -0.615 & $0.044^{*}$ \\
\hline & & & Magnetic & -0.758 & $0.007^{*}$ & -0.647 & $0.031 *$ \\
\hline & \multirow{4}{*}{$80 \mathrm{~mA}$} & \multirow{2}{*}{$\mathrm{X}$ axis } & Electrical & -0.857 & $0.001 *$ & -0.802 & $0.003^{*}$ \\
\hline & & & Magnetic & -0.715 & $0.013^{*}$ & -0.076 & 0.824 \\
\hline & & \multirow{2}{*}{ Y axis } & Electrical & -0.735 & $0.010^{*}$ & 0.089 & 0.795 \\
\hline & & & Magnetic & -0.627 & $0.039^{*}$ & 0.310 & 0.353 \\
\hline
\end{tabular}




\begin{tabular}{|l|l|l|l|l|l|l|l|}
\hline & \multirow{2}{*}{$Z$} & axis & Electrical & -0.843 & $0.001^{*}$ & 0.219 & 0.517 \\
\cline { 4 - 8 } & & Magnetic & -0.326 & 0.328 & -0.087 & 0.799 \\
\hline
\end{tabular}

* Significant correlation P-value $<0.05$

As presented at table (8), the correlations between distance and the mean value of electrical and magnetic fields around the Diadynamic MF electrodes at different intensities and axes at earthed and unearthed were studied through the Pearson product moment correlation coefficient.

Table (8): The statistical analysis of (Correlation between Distance and electrical or magnetic current in Diadynamic MF electrodes) in both Earthed and Un Earthed conditions:

\begin{tabular}{|c|c|c|c|c|c|c|c|}
\hline & \multirow[b]{2}{*}{ Intensity } & \multirow[b]{2}{*}{ Axis } & \multirow[b]{2}{*}{ Current } & \multicolumn{2}{|l|}{ Earthed } & \multicolumn{2}{|l|}{ Un-Earthed } \\
\hline & & & & $\begin{array}{l}\text { Pearson } \\
\text { Correlation }\end{array}$ & P-value & $\begin{array}{l}\text { Pearson } \\
\text { Correlation }\end{array}$ & P-value \\
\hline \multirow{18}{*}{ Electrodes } & \multirow{6}{*}{$10 \mathrm{~mA}$} & \multirow{2}{*}{$\mathrm{X}$ axis } & Electrical & -0.758 & $0.007^{*}$ & -0.664 & $0.026^{*}$ \\
\hline & & & Magnetic & -0.615 & $0.044^{*}$ & -0.763 & $0.006^{*}$ \\
\hline & & \multirow{2}{*}{$\mathrm{Y}$ axis } & Electrical & -0.895 & $0.000^{*}$ & -0.898 & $0.000^{*}$ \\
\hline & & & Magnetic & -0.620 & $0.042^{*}$ & -0.191 & 0.574 \\
\hline & & \multirow[t]{2}{*}{$\begin{array}{|ll|}\mathrm{Z} & \text { axis } \\
\end{array}$} & Electrical & -0.020 & 0.953 & 0.237 & 0.483 \\
\hline & & & Magnetic & -0.667 & $0.025^{*}$ & -0.924 & $0.000 *$ \\
\hline & \multirow{6}{*}{$50 \mathrm{~mA}$} & \multirow{2}{*}{$\mathrm{X}$ axis } & Electrical & -0.912 & $0.000^{*}$ & -0.840 & 0.001* \\
\hline & & & Magnetic & -0.745 & $0.008^{*}$ & \begin{tabular}{|l|}
-0.697 \\
\end{tabular} & $0.017 *$ \\
\hline & & \multirow{2}{*}{$\mathrm{Y}$ axis } & Electrical & -0.633 & $0.036^{*}$ & -0.737 & $0.010 *$ \\
\hline & & & Magnetic & -0.447 & 0.168 & -0.635 & $0.036^{*}$ \\
\hline & & \multirow[t]{2}{*}{$\mathrm{Z}$ axis } & Electrical & -0.421 & 0.197 & 0.503 & 0.115 \\
\hline & & & Magnetic & -0.326 & 0.328 & -0.729 & 0.011* \\
\hline & \multirow{6}{*}{$80 \mathrm{~mA}$} & \multirow{2}{*}{$\mathrm{X}$ axis } & Electrical & -0.908 & $0.000 *$ & -.911 & $0.000 *$ \\
\hline & & & Magnetic & -0.720 & $0.013^{*}$ & -0.706 & $0.015^{*}$ \\
\hline & & \multirow{2}{*}{$\mathrm{Y}$ axis } & Electrical & -0.591 & 0.056 & -0.305 & 0.362 \\
\hline & & & Magnetic & -0.783 & $0.004^{*}$ & -0.702 & $0.016^{*}$ \\
\hline & & \multirow{2}{*}{$\mathrm{Z}$ axis } & Electrical & -0.861 & $0.001 *$ & 0.082 & 0.812 \\
\hline & & & Magnetic & -0.836 & $0.001 *$ & \begin{tabular}{|l|}
-0.429 \\
\end{tabular} & 0.189 \\
\hline
\end{tabular}

* Significant correlation P-value $<0.05$

\section{Discussion}

In the present study, the maximum values recorded for electric field intensity $(\mathrm{V} / \mathrm{m})$ with interferential bipolar technique were below the limits of guidelines of ICNIRP. However, the magnetic flux density values (Gauss) that were measured at the electrodes were 4.80, 4.43 and $1.13 \mathrm{G}$ at the electrodes and 2.40, 2.77 and $2.40 \mathrm{G}$ at 1 meter from the electrodes in $\mathrm{x}, \mathrm{y}$ and $\mathrm{z}$ planes respectively, under ungrounded condition. These values are higher than the safe limit set by ICNIRP guidelines ( $1 \mathrm{G}$ for occupational exposure and $0.27 \mathrm{G}$ for public exposure). Moreover, the mean measured values for electric field intensity (V/m) with interferential quadripolar technique were below the limits of guidelines of ICNIRP, however the magnetic flux density values (Gauss) that measured at the electrodes were higher $(2.33,4.27$ and $1.80 \mathrm{G}$ at the electrodes in $\mathrm{x}, \mathrm{y}$ and $\mathrm{z}$ planes respectively, under ungrounded conditions) than the reference values set by ICNIRP guidelines ${ }^{9}$.It was observed that the magnetic flux density values recorded at the electrodes of interferential and diadynamic techniques decreased at a distance of 1 meter, but they were still higher than the reference values set by ICNIRP guidelines for occupational and public exposure. This means that part of the energy leaks to the therapist and affects him, so the distance between the therapist and device's electrodes especially must be increased than one meter. In line with this finding, Shah and Farrow ${ }^{12}$ reported that Physiotherapists' professional guidelines should revise and increase the current safe distance of $1 \mathrm{~m}$ from an operating short wave device (SWD). They have concluded that the revised safe distance should be at least $2 \mathrm{~m}$ for CSWD and $1.5 \mathrm{~m}$ for PSWD.The present work illustrated that all values of magnetic flux density (Gauss) that were measured and recorded on the electrodes were lowered to the safe limits in both interferential and diadynamic techniques when the device was connected to the ground electrode. Equipment grounding ensures that in the event of a fault state, the worker will not be exposed to electric shock when contacting the device. Additionally, in the absence of proper grounding of the 
equipment, the electric charges will be accumulated on the metal parts of the device. When the accumulated charges exceed the limit, the collected energy will be emitted from the equipment surface as an electromagnetic field ${ }^{13}$. So proper grounding of the equipment should contribute to the reduction of the amount of electric and magnetic field exposure near or around the equipment.Taken together the magnetic flux density values surrounding ungrounded physical therapy equipment can't be neglected as it may lead to many health problems for the physiotherapist, assistants, and patient companions. The epidemiological studies have consistently found that everyday chronic low-intensity magnetic field exposure is associated with an increased risk of childhood leukemia. IARC ${ }^{14}$ has classified such fields as possibly carcinogenic ${ }^{15}$.

Conflicts of interest : None.

\section{References}

1. Dewan A, Sharma R. Effectiveness of transcutaneous electrical nerve stimulation and interferential electrotherapy in adhesive capsulitis. Punjab Journal of Orthopaedics2011;12(1):64-71.

2. Nussbaum E, Rush P, Disenhaus L. The effects of interferential therapy on peripheral blood flow, Physical Therapy, 1990;76-803.

3. Fish D. Effect of anodal high voltage pulsed current on edema formation in frog hind limbs. Physical Therapy, 1991; 71:724-733. doi:10.1093/ptj/71.10.724.

4. Kitchen S, Bazin S. Electrotherapy: Evidence-Based Practice. 11th ed. Churchill Livingstone, London 2002.

5. Low J, Reed A. Electrotherapy explained principles and practice. Third edition, ButterworthHeinemann, Oxford. 2002.

6. .Prentice WE. Therapeutic modalities: for sports medicine and athletic training. 6th ed. McGraw-Hill Higher Education, New York 2009.

7. Camargo BF, Dos Santos, MM, Liebano RE." Hypoalgesic effect of Bernard's diadynamic currents on healthly individuals".RevistaDorSao Paulo2012; 13(4):327-31. doi:10.1590/S180600132012000400004

8. Den Adel RV, Luykx RHJ. "Low and medium Frequency Electrotherapy", Enraf-Nonius B.V. Rotterdam, 1480.(2005);762-43;pp12-13 .

9. International Committee for Non-Ionizing Radiation Protection (ICNIRP). Guidelines for limiting exposure to time-varying electric and magnetic fields (1 Hz to $100 \mathrm{kHz})$. Health Physics2010; 99(6):818-836.

10. Ubeda A, Martinez MA, Cid MA, Chacon L, Trillo MA, Leal J. Assessment of Occupational Exposure to Extremely Low Frequency Magnetic Fields in Hospital Personnel. Bioelectromagnetics2011;32:378387. doi:10.1002/bem.20644

11. Australian Radiation Protection and Nuclear Safety Agency (ARPANSA). Radiation Protection Standards, Maximum Exposure Levels to Radiofrequency Fields $3 \mathrm{~K} \mathrm{~Hz}$ to $300 \mathrm{G} \mathrm{Hz}$. Commonwealth of Australia 2002.

12. Shah SGS, Farrow A. Assessment of Physiotherapists' Occupational Exposure to Radiofrequency Electromagnetic Fields from Shortwave and Microwave Diathermy Devices: A Literature Review. Journal of Occupational and Environmental Hygiene, 2013; 10(6):312-327. doi:10.1080/15459624.2013.782203

13. Pease WS, Grove SL. Electrical safety in electrodiagnosticmedicine. PM R 2013;5(5 Suppl):S8-13. 34.

14. International Agency for Research on Cancer (IARC). IARC classifies radiofrequency electromangetic fields as possible carcinogen to humans. Lyon (France): IARC; http://www.iarc.fr/en/mediacentre/pr/2011/pdfs/pr208_E.pdf2011.

15. World Health Organization (WHO). Electromagnetic fields and public health: exposure to extremely low frequency fields. Fact Sheet No 322. Geneva: World Health Organization2007. 